Typeset with jpsj2.cls $<$ ver.1.1 $>$

\title{
Numerical study of the lattice vacancy effects on the single-channel electron transport of graphite ribbons
}

\author{
Katsunori WAKABAYASHI ${ }^{1,2} *$ \\ ${ }^{1}$ Department of Quantum Matter Science, Graduate School of Advanced Sciences of Matter (ADSM), Hiroshima \\ University, Higashi-Hiroshima 739-8527 \\ ${ }^{2}$ Department of Physical Electronics, Faculty of Engineering, Hiroshima University, Higashi-Hiroshima 739-8527
}

(Received June 5, 2021)

\begin{abstract}
Lattice vacancy effects on electrical conductance of nanographite ribbon are investigated by means of the Landauer approach using a tight binding model. In the low-energy regime ribbons with zigzag boundary provide a single conducting channel whose origin is connected with the presence of edge states. It is found that the chemical potential dependence of conductance strongly depends on the difference $(\Delta)$ of the number of removed $\mathrm{A}$ and $\mathrm{B}$ sublattice sites. The large lattice vacancy with $\Delta \neq 0$ shows $2 \Delta$ zeroconductance dips in the single-channel region, however, the large lattice vacancy with $\Delta=0$ has no dip structure in this region. The connection between this conductance rule and the Longuet-Higgins conjecture is also discussed.
\end{abstract}

KEYWORDS: Nanographite, graphite ribbon, lattice vacancy, zero-conductance, Landauer formula

\section{INTRODUCTION}

Recently, nanometer-sized carbon systems such as carbon nanotube, ${ }^{1,2)}$ fullerene molecule and nanographite have attracted much attention by the possibilities for the realization of carbon-based molecular-electronic devices. In these systems, the geometry of $\mathrm{sp}^{2}$ carbon networks has much influence on the electronic states near the Fermi level. Not only the closed carbon molecules such as carbon nanotubes and fullerene molecules, but also systems with open boundaries also display unusual features connected with their boundaries. The existence of graphite edges strongly affects the $\pi$-electronic states in nanometer-sized graphite fragments (nanographites). ${ }^{3)}$ There are two basic edge shapes in graphite, armchair and zigzag. For the model of graphite ribbons, one-dimensional graphite lattices of finite width, ${ }^{3,9,10)}$ it was shown that ribbons with zigzag edges (zigzag ribbon) possess localized edge states with energies close to the Fermi level. ${ }^{3,9,10}$ ) These edge states correspond to the non-bonding molecular orbitals as can be seen by examining the analytic solution for semi-infinite graphite with a zigzag edge. In contrast, edge states are completely absent for ribbons with armchair edges. We have also pointed out that the edge states play important roles in magnetic properties in nanometer-sized graphite systems, because of their relatively large contribution to the density of states at the Fermi energy. ${ }^{3,9-11)}$ In this paper, we study the impurity scattering effect and the lattice vacancy effect in the electron transport of zigzag nanographite

\footnotetext{
*Email address: waka@qp.hiroshima-u.ac.jp
} 
ribbons, in which the conductance crucially depends on the geometry of the lattice defects. We find that the conductance rule: the large lattice vacancy with $\Delta \neq 0$ shows $2 \Delta$ zero-conductance dips in the single-channel region, however, the large lattice vacancy with $\Delta=0$ has no dip structure in that region.

\section{ELECTRONIC PROPERTIES OF NANOGRAPHITE RIBBONS}

Let us introduce the electronic states of nanographites based on the tight binding model. The tight-binding Hamiltonian is defined by

$$
H=\sum_{i, j} t_{i, j}|i\rangle\left\langle j\left|+V \sum_{\alpha}\right| \alpha\right\rangle\langle\alpha|
$$

where $t_{i, j}=-t$ if $i$ and $j$ are nearest neighbors, otherwise 0 , and $|i\rangle$ is a localized orbital on site $i$. The second term represents the impurity potential, $\mathrm{V}$ and $\alpha$ are the strength of the impurity potential and the location of impurity, respectively. In Fig.1(a), the graphite ribbon with zigzag edges (zigzag ribbons) is shown. We assume all edge sites are terminated by H-atoms. The ribbon width $N$ is defined by the number of zigzag lines. As the graphite lattice is bipartite, the $\mathrm{A}(\mathrm{B})$-site on the $\mathrm{n}$-th zigzag line is called $\mathrm{nA}(\mathrm{nB})$-site. Fig. 1(b) is the energy band structure of zigzag ribbon for $N=20$. The zigzag ribbons are metallic for all $N$. One of the remarkable features is the appearance of partly flat bands at the $\operatorname{Fermi} \operatorname{level}(E=0)$, where the electrons are strongly localized near zigzag edges. ${ }^{3)}$

The analytic solution of the partly flat band for a semi-infinite graphite sheet with a zigzag edge ( $1 \mathrm{~A}$ and $1 \mathrm{~B}$ site shall be the edge sites and $\mathrm{N}$ is infinite in Fig.1(a)) can be expressed as $\phi_{n A}=D_{k}^{n-1}$ and $\phi_{n B}=0$, where $\phi_{n A}\left(\phi_{n B}\right)$ means the amplitude of the edge states on $\mathrm{nA}(\mathrm{nB})$ site and $D_{k}=-2 \cos (k / 2)$. It is worth noting that the edge state has a non-zero amplitude only on one sublattice, i.e. non-bonding character. Because of the convergence condition of the edge states, the wave number $k$ must be $2 \pi / 3 \leq k \leq \pi$, where $\left|D_{k}\right| \leq 1$. In this $k$-region, the edge states make a flat band at $\mathrm{E}=0$ (Fermi energy). It should be noted that at $k=\pi$ the edge states are perfectly localized at the $1 \mathrm{~A}$ sites, but at $k=2 \pi / 3$ the edge states are completely delocalized. However, when we consider the zigzag ribbons, two edge states which come from both sides will overlap each other and cause the bonding and anti-bonding splitting. The magnitude of the overlap becomes larger when the wave number approaches $2 \pi / 3$, because the penetration length of the edge states gets larger there. Therefore, the partly flat bands have a slight dispersion, which leads to one channel for the electron transport. The energy region of the single-channel, $\Delta_{z}$, ( defined by the energy gap to the next channel) is approximately, $\Delta_{z}=-4 t \cos [(N-1) \pi /(2 N+1)]$.

In this manuscript, we evaluate the electrical conductance by the Landauer formula, ${ }^{12)}$

$$
G(E)=\frac{e^{2}}{\pi \hbar} T(E)
$$


where $T$ is the transmission probability through the impurity potential. We use the recursive Green function method for the calculation of the transmission probability, ${ }^{13)}$ which provides high numerical accuracy and efficiency. We also use this method to calculate the electron waves and electric current around impurity. ${ }^{14,16)}$

\section{SINGLE IMPURITY SCATTERING}

In this section, we study the single impurity scattering effect in the nanographite ribbons. In the low-energy single-channel region, even the existence of a single-impurity causes the the nontrivial behavior to the position and strength dependence of the impurity due to the edge localized nature of the edge states.

Before we consider the electron transmission through an impurity or lattice vacancy in zigzag ribbon, let us discuss the single impurity problem in a graphite sheet. The problem described here is the electron scattering by the $\delta$-function type impurity potential with the strength $V$, at an A-site $(\boldsymbol{r}=0)$. The Schrödinger equation is written as

$$
\left(\begin{array}{cc}
V \delta(\mathbf{r}) & \epsilon_{k}^{*} \\
\epsilon_{k} & 0
\end{array}\right)\left(\begin{array}{c}
\phi_{\mathrm{A}}(\mathbf{r}) \\
\phi_{\mathrm{B}}(\mathbf{r})
\end{array}\right)=E\left(\begin{array}{c}
\phi_{\mathrm{A}}(\mathbf{r}) \\
\phi_{\mathrm{B}}(\mathbf{r})
\end{array}\right),
$$

where $\epsilon_{k}=-t\left(\exp \left(-i k_{y} a\right)+\exp \left(i\left(\sqrt{3} k_{x}+k_{y}\right) a / 2+\exp \left(-i\left(\sqrt{3} k_{x}-k_{y}\right) a / 2\right)\right.\right.$ describes the electron hopping from the A site to the $\mathrm{B}$ site in the unit cell. Here we take the translational invariant direction of the zigzag axis as the $\mathrm{x}$-axis, the $\mathrm{y}$-axis is perpendicular to the $\mathrm{x}$-axis, and $a$ is the lattice constant. Following the standard approach by Koster and Slater, ${ }^{17)}$ we obtain the self-consistent equation determining the impurity state energy, $E$, as follows,

$$
\frac{1}{V}=\frac{E}{(2 \pi)^{2}} \int_{1 \mathrm{stBZ}} d \boldsymbol{k} \frac{1}{E^{2}-\left|\epsilon_{k}\right|^{2}},
$$

where the integral is taken over the 1st Brillouin zone of the graphite sheet. This integral is in general complex number, which means the bound state has a resonant nature, i.e. virtual bound state with finite lifetime. However, in the limit of infinity $V$, i.e. a single vacancy in the graphite sheet, the virtual bound state becomes well-defined state bound to the impurity, and the energy level of the impurity state comes to $E=0$. We should note that this resonant behavior is quite similar to the single impurity problem in a $d$-wave superconductor, ${ }^{18)}$ because of the finite density of states $D(E) \propto E$ in both systems. The wavefunction then can be written in $\boldsymbol{k}$-space as

$$
\begin{aligned}
\phi_{A k} & =\frac{V}{L^{2}} G_{A}^{0} \frac{E}{E^{2}-\left|\epsilon_{k}\right|^{2}} \\
\phi_{B k} & =\frac{V}{L^{2}} G_{A}^{0} \frac{\epsilon_{k}}{E^{2}-\left|\epsilon_{k}\right|^{2}},
\end{aligned}
$$

where $G_{A}^{0}=\sum_{k} \phi_{A k}$. From the normalization of these wavefunctions, we can find that, in the limit of infinity $V, \phi_{A}(\boldsymbol{r})=0$ and $\phi_{B}(\boldsymbol{r}) \neq 0$, i.e. non-bonding character. Thus if we make a 
single vacancy at an $\mathrm{A}(\mathrm{B})$ site in a graphite sheet, a vacancy bound state appears at $E=0$ and its wavefunction has a non-bonding character, where the amplitudes of the $\mathrm{A}(\mathrm{B})$-sites are zero.

In Fig.2, we show the Fermi energy dependence of the transmission probability in the singlechannel energy region for the graphite ribbon with $N=30$, where the impurity is located at center of the ribbon (15B site). The strength of the impurity potential is changed by $V / t=1,10^{1}, 10^{2}, 10^{3}$. The conductance for negative $V / t$ can be obtained by the transformation of $E \rightarrow-E$ in Fig.2. We find pronounced conductance dip structures for sufficiently strong impurity potential. At the energies of conductance dips, the electron waves are localized around the impurity site. Thus the conductance dips is caused by virtual bound states at the impurity. We see also that the phase jumps of the transmission coefficient by $\pi$ at the energy of dips. The $\pi$-phase jump guarantees the perfect reflection at the conductance dip. ${ }^{14}$ ) The weak impurity potential (less than $t$ ) does not have much influence on the conductance in the single-channel region, although the graphite ribbon is one-dimensional system. The width of zero-conductance resonance means the inverse of the traversal time that the electrons pass through the impurity region. In many cases, the traversal time increases with apart from $E=0$, because the group velocity of edge states gets larger with leaving from $E=0$. Thus the conductance drastically changes by the strength of impurity potential.

In Fig.3, we show the dependence of the position of zero-conductance dip on the strength of impurity potential. In the limit of $V / t \rightarrow \pm \infty$, the impurity level approaches $E=0$, where a single $\mathrm{nA}(\mathrm{B})$-site impurity produces a non-bonding state so that the amplitude of $\mathrm{nA}(\mathrm{B})$-sites is zero. On the other hand, the edge states has the character that electrons strongly localized on $\mathrm{nA}(\mathrm{nB})$-sites for $\mathrm{n}<N / 2(\mathrm{n}>N / 2)$. Therefore the impurity state caused by the impurity potential located at $\mathrm{nA}$-site $(\mathrm{n}<N / 2)$ strongly couples with the edge states, resulting in the splitting of the level of zeroconductance $\operatorname{dips}($ Fig.3(a)). However, the impurity state caused by the impurity potential located at $\mathrm{nB}$-site $(\mathrm{n}<N / 2)$ cannot couple with the edge states because the impurity states and edge states live on the same sublattice sites, so that zero-conductance dip appears only near $E=0$ (Fig.3(b)) in the strong $V / t$ limit. Note that nB-site impurity does not produce zero-conductance dips in weak $V / t$ region, and nA-site impurity produces a single zero-conductance dip close to $E=0$ in weak $V / t$ region.

The Fermi energy dependence of the transmission through a single vacancy $(V / t \rightarrow \infty)$ in zigzag ribbon is shown in Fig.4. The position of the vacancy site is denoted in the figure. In this limit, two zero-conductance dips appear with mirror symmetry to $E=0$. Since a single vacancy on $\mathrm{nA}(\mathrm{B})$-site produces a zero-energy non-bonding state with zero-amplitude on the $\mathrm{nA}(\mathrm{B})$-sites, the non-bonding states at the vacancy and one of the two edges couple forming a bonding and antibonding configuration with energy level below and above zero causing the zero-conductance dips at the corresponding energies. The vacancy state due to $\mathrm{nB}$-site vacancy for $\mathrm{n}<N / 2$ are so far removed from the corresponding edge that their overlap is very small and the corresponding zero- 
conductance dips occur at energy extremely close to $E=0$. Thus, in the low-energy single-channel region, the electric conductance shows the non-trivial behavior, even for the single impurity case, due to the edge localized non-bonding nature of the edge states and non-bonding character of the impurity states.

Here we briefly discuss the origin of the zero-conductance dips observed. Each zero-conductance resonance can be associated with a quasi-bound state around the vacancy or the impurity, yielding the formation of standing waves. Around the energies of the each zero-conductance resonances, the electric currents show the Kekulé-like current vortex pattern, which can be observed in the nanographite ribbon junctions. ${ }^{14,16)}$ We have also checked each resonance feature can be characterized by a zero-pole pair in the complex energy plane. This suggest that the zero-conductance dip corresponds to a Fano resonance which is known to occur when two scattering channels are available, one corresponding to a continuum of states and the other to a discrete quasi-bound state. $^{19,20)}$

\section{LARGE SIZE LATTICE VACANCY}

Next we study the effect of large size lattice vacancies. Examples of large size lattice vacancy are shown in Fig.5, where the removed sites are denoted by black and white circles. There are two typical lattice vacancies, (i) site-centered vacancies (Fig.5(a)) and (ii) ring-centered vacancies (Fig.5(b)). They have three- and six-fold symmetry, respectively. The notation S-m (or R-m) denotes the site(ring)-centered lattice vacancy with $\mathrm{m}$ removed sites. The difference $\Delta=\left|N_{A}-N_{B}\right|$ is always zero for ring-centered vacancy, where $N_{A}\left(N_{B}\right)$ is the number of removed A (B) sublattice sites.

The conductance of zigzag ribbon $(\mathrm{N}=20)$ with site-centered vacancies is shown in Fig.6 for the case of (a) S-13 and (b) S-10. The indices in the figure mean the center position of the vacancy. The conductance is very sensitive to the position of vacancy, similar to the single-site vacancy case. In analogy to the Longuette-Higgins(LH) conjecture, ${ }^{21)}$ the vacancy with $\Delta \neq 0\left(N_{A}<N_{B}\right)$ produces $\Delta$-degenerate non-bonding vacancy states where the amplitude of nB-sites is zero. Therefore, the S-13 vacancy $\left(\Delta\left(=N_{B}-N_{A}\right)=1\right)$ produces a non-bonding vacancy state, where the $\mathrm{nB}$ sites are node, and couples with the edge state which comes from the upper edge in Fig. 1(a). The case of the S-10 vacancy which is $\Delta=4$ produces, however, many more zero-conductance dips, with increasing the strength of coupling between the edge states and vacancy states. Therefore it is considered that, if the large size single vacancy with $\Delta \neq 0$ such as the Fig.5 is introdunced in a graphite sheet, the $\Delta$-degenerated vacancy bound states are produced at $E=0$.

In Fig.6 (c), the Fermi energy dependence of the conductance of zigzag ribbon $(\mathrm{N}=20)$ with a ring-centered vacancy is shown. The conductance gradually decreases with increasing the size of the vacancy, however no dip structure appears in the single-channel region. The conductance dips 
appear only around the energy where the channel number changes. Since these dips are caused by the multi-channel scattering effects, we do not pay attention to these dip structures in this paper. Since in analogy to the LH conjecture, the vacancy with $\Delta=0$ does not create non-bonding vacancy states, the zero-conductance resonances do not appear in the single-channel energy region. Interestingly, the conductance is not sensitive to the position of ring-centered vacancy. The resonant tunneling-like behavior around $E=0$ is mimic, and is caused by the variation of the group velocity, because the group velocity rapidly decreases with approaching to $E=0$ due to the partly flat band. It should be noted that the transmission probability is exactly zero and singular at $E=0$, because the group velocity is zero at $E=0$.

Thus our numerical results show that the large size vacancy with $\Delta \neq 0$ produces $2 \Delta$ zeroconductance dips in the single-channel energy region, however, the large size vacancy with $\Delta=0$ has no zero-conductance dip in the single-channel region. This rule is not strict, so that there are a few exceptions which do not satisfy the rule because of the complicated multiple-scattering effects between edge states and vacancy states. However, most of the vacancies numerically tested satisfy this conductance rule. Furthermore, it should be noted that this conductance rule implies the following rule: the large size vacancy with $\Delta \neq 0$ in a graphite sheet produces the $\Delta$-degenerated zero-energy non-bonding bound states and the vacancy with $\Delta=0$ in a graphite sheet do not produce the bound state. Thus this rule has the negative-positive relation with the LH-rule discussed in a finite molecule. A rigorous proof remains a problem for future study.

\section{CONCLUSION}

In conclusion, we have studied the effects of a non-magnetic impurity on electrical conductance of graphite ribbons with zigzag edges. The conductance strongly depends on the position and strength of the impurity. For large size lattice vacancy, the behavior of the conductance drastically changes with the difference $(\Delta)$ of removed $\mathrm{A}$ and $\mathrm{B}$ sublattice sites. For the $\Delta \neq 0$ case, the Fermi energy dependence of conductance shows dip structures. For the $\Delta=0$ case, conductance dips appear only around the energies where the number of channel changes, but no conductance dips in the single channel region. Our results support that the lattice vacancy with $\Delta \neq 0$ produces the $\Delta$-non-bonding vacancy bound states, however the vacancy with $\Delta=0$ do not produce bound state. Our work shows that the impurity effects in nano-graphite ribbons are quite different from the case of usual quantum wires ${ }^{23)}$ and carbon nanotubes. ${ }^{22)}$ In this paper, we have pointed out that the electric conductance through the nanographite ribbons with a single vacancy produces the pronounced zero-conductance resonances and shows crucial dependence on the vacancy geometry and the strength of the impurity potential. Therefore the nanographite ribbons which posses many vacancy or impurity potential show the non-trivial behavior in the electrical conductance. We demonstrate this problem elsewhere. 


\section{Acknowledgments}

I would like to thank M. Sigrist, M. Igami and T. Kawabe for their many helpful discussions. I am grateful for support by Grant-in-Aid for Scientific Research from Ministry of Education, Science and Culture, Japan, and the financial support from the Foundation Advanced Technology institute and from the Kinki-chihou Hatsumei Center. Numerical calculations were performed in part in Institute for Molecular Science.

1) M. S. Dresselhaus, G. Dresselhaus, and P. C. Eklund, Science of Fullerenes and Carbon Nanotubes, (Academic Press, San Diego, 1996).

2) R. Saito, G. Dresselhaus, and M. S. Dresselhaus, Physical Properties of Carbon Nanotubes, (Imperial College Press, London, 1998).

3) M. Fujita, K. Wakabayashi, K. Nakada and K. Kusakabe, J. Phys. Soc. Jpn. 65, 1920 (1996)

4) Note that graphite ribbons can be a simple model of defective carbon nanotubes of carpet-roll or papier-mâché forms, ${ }^{5)}$ activated carbon fibers ${ }^{6)}$ and nanographites. ${ }^{7)}$ There are also some trials to fabricate nanographite ribbons on metal substrates. ${ }^{8}$

5) O. Zhou, R. M. Fleming, D. W. Murphy, C. H. Chen, R. C. Haddon, A. P. Ramirez, and S. H. Glarum, Nature 263, 1744 (1994); S. Amelinckx, D. Bernaerts, X. B. Zhang, G. V. Tendeloo, and J. V. Landuyt, Nature 267, 1334 (1995).

6) A. Nakayama, K. Suzuki, T. Enoki, S. L. di Vittorio, M. S. Dresselhaus, K. Koga, M. Endo and N. Shindo, Synth. Met. 55-57, 3736 (1993)

7) O. E. Andersson, B. L. V. Prasad, H. Sato, T. Enoki, Y. Hishiyama, Y. Kaburagi, M. Yoshikawa, S. Bandow, Phys. Rev. B 58, 16387 (1998).

8) M. Terai, N. Hasegawa, M. Okusawa, S. Otani, and C. Oshima, Appl. Sur. Sci. 130-132, 876 (1998); C. Oshima, private communication.

9) K. Nakada, M. Fujita, G. Dresselhaus and M. S. Dresselhaus, Phys. Rev. B 54, 17954 (1996).

10) K. Wakabayashi, M. Fujita, H. Ajiki, and M. Sigrist, Phys. Rev. B 59, 8271 (1999).

11) K. Wakabayashi, M. Fujita, and M. Sigrist, Phys. Soc. Jpn. 672089 (1998).

12) M. Büttiker, Y. Imry, R. Landauer, and S. Pinhas, Phys. Rev. B 31, 6207 (1985).

13) A. MacKinnon, Z. Phys. B - Condensed Matter 59385 (1985); T. Ando, Phys. Rev. B 448017 (1991).

14) K. Wakabayashi, and M. Sigrist, Phys. Rev. Lett. 84, 3390 (2000).

15) The zigzag (bearded) ribbons at $k=\frac{2 \pi}{3 a}$ can be mapped to a finite one-dimensional chain with $2 N(2 N+1)$ sites, which can be solved analytically (see the appendix of ${ }^{10)}$ ). From this analytic solution, the form of $\Delta_{z}\left(\Delta_{b}\right)$ is easily derived.

16) K. Wakabayashi, Phys. Rev. B., 64125428 (2001).

17) G. F. Koster, and J. C. Slater, Phys. Rev. 95, 1167 (1954); G. F. Koster, Phys. Rev. 95, 1436 (1954); G. F. Koster, and J. C. Slater, Phys. Rev. 96, 1208 (1954).

18) A. V. Balatsky, M. I. Salkola, and A. Rosengren, Phys. Rev. B 51, 15547 (1995).

19) Z. Shao, W. Porod, and C. S. Lent, Phys. Rev. B49, 7453 (1994).

20) W. Porod, Z. Shao, and C. S. Lent, Phys. Rev. B48, 8495 (1993); W. Porod, Z. Shao, and C. S. Lent, Appl. Phys. Lett. 61, 1350 (1992).

21) H.C. Longuet-Higgins, J. Chem. Phys. 18, 265 (1950).

22) M. Igami, T. Nakanishi, and T. Ando, J. Phys. Soc. Jpn. 683146 (1999).

23) P. F. Bagwell, Phys. Rev. B 41, 10354 (1990). 
(a)

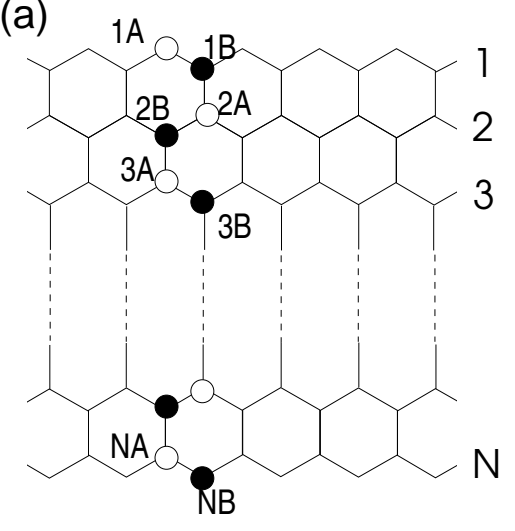

(b)

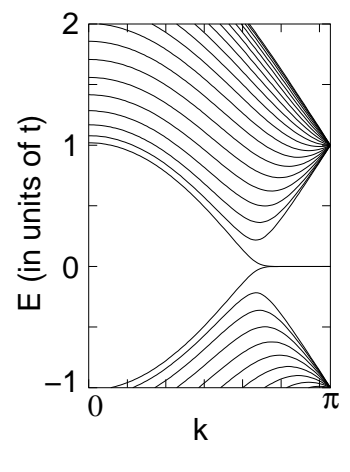

Fig. 1. (a) Graphite ribbon with zigzag edges (zigzag ribbon). (b) Energy band structure of zigzag ribbon (N=20).

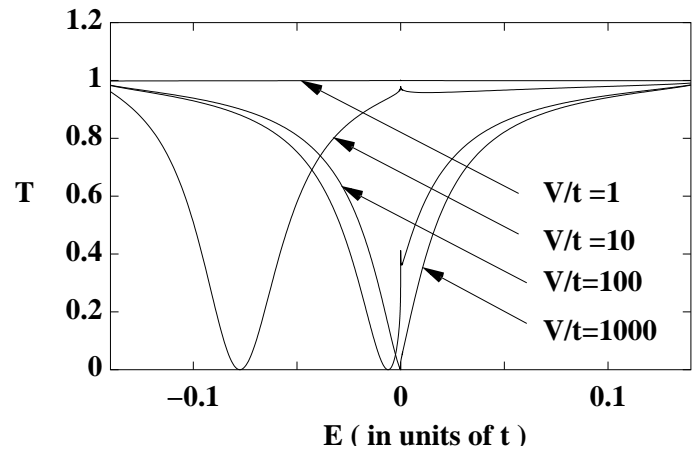

Fig. 2. Fermi energy dependence of the transmission probability for the various strength of impurity potential $(\mathrm{V} / t=$ $1,10^{1}, 10^{2}, 10^{3}$ ) when the impurity is located at $15 \mathrm{~B}$ site of the zigzag ribbon with $\mathrm{N}=30$.

(a)

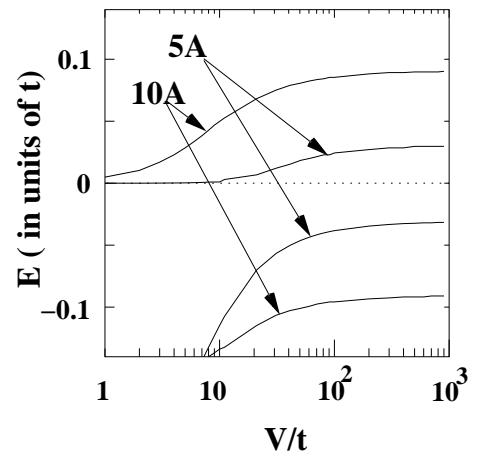

(b)

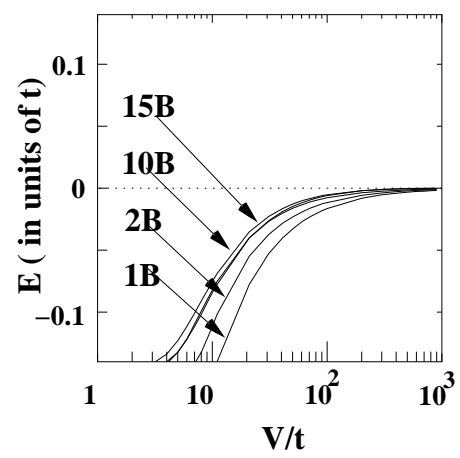

Fig. 3. The dependence of the position of zero-conductance dips on the strength of impurity potential (a) for $5 \mathrm{~A}$ and $10 \mathrm{~A}$ site, (b) for $1 \mathrm{~B}, 2 \mathrm{~B}, 10 \mathrm{~B}$ and $15 \mathrm{~B}$. 


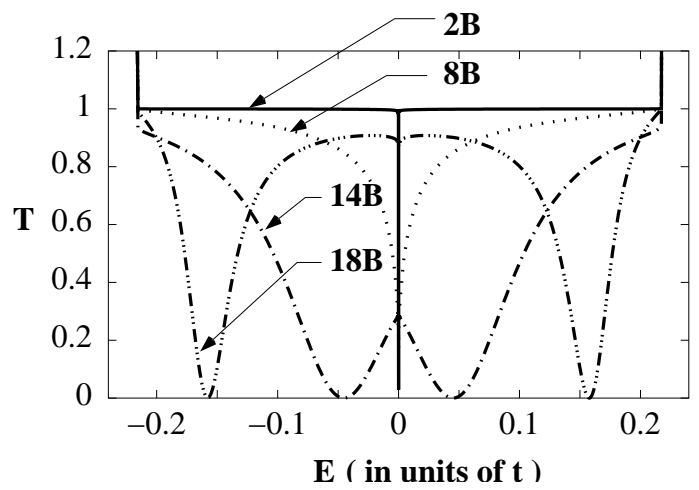

Fig. 4. Fermi energy dependence of the transmission probability of zigzag ribbon $(\mathrm{N}=20)$ with a single-site vacancy.

The position of the vacancy site is denoted in the figure.

(a)

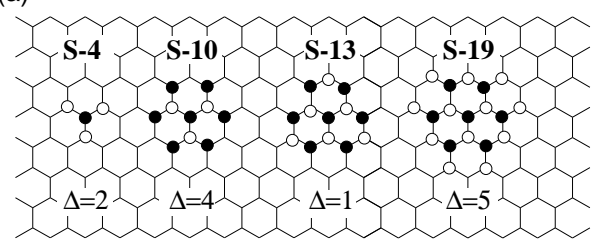

(b)

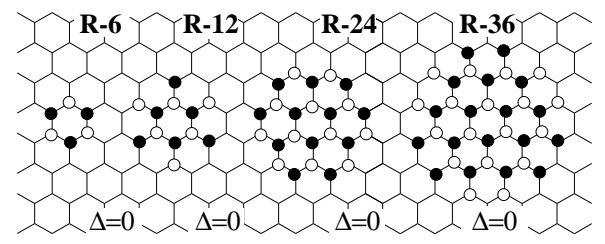

Fig. 5. Examples of (a) site-centered and (b) ring-centered vacancies, and $\Delta$ denotes the difference of the number of removed $\mathrm{A}$ and $\mathrm{B}$ sublattice sites.

(a)

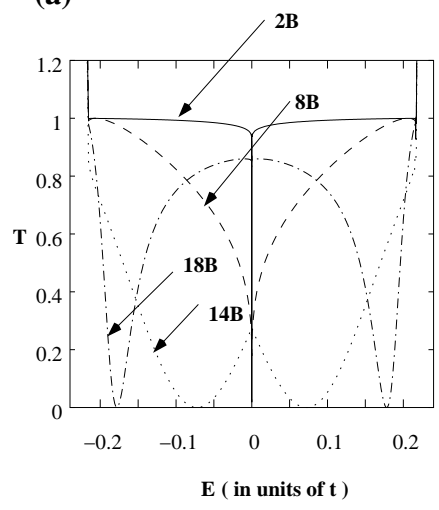

(b)

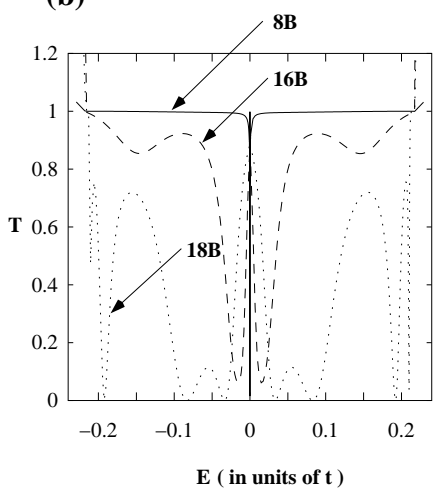

(c)

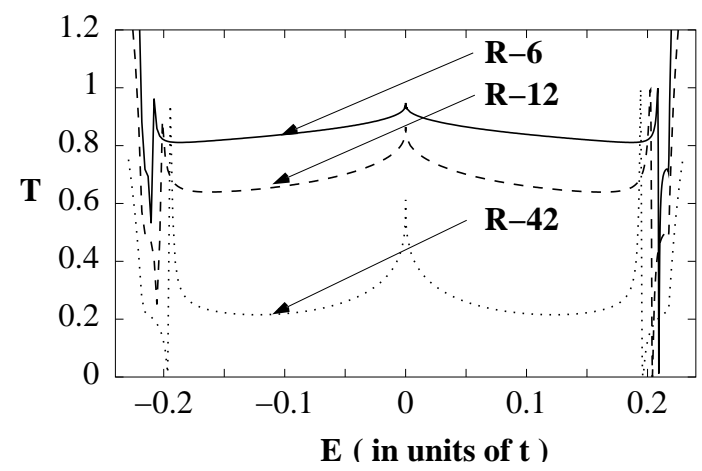

Fig. 6. Fermi energy dependence of the transmission probability for the site-centered vacancies of (a) S-13 and (b) S-10, where the indices denote the center position of the vacancy. (c) The transmission probability for ring-centered vacancies of R-6, R-12 and R-42, which are located at the center of the ribbon. 